\title{
JNK signaling pathway is involved in piperlongumine-mediated apoptosis in human colorectal cancer HCT116 cells
}

\author{
WEN LI $^{1-4 *}$, CHUANGYU WEN ${ }^{1-3^{*}}$, HAIYAN BAI ${ }^{5}$, XIAOYAN WANG $^{1-3}$, XIAOLI ZHANG $^{1}$, \\ LANLAN HUANG ${ }^{1-3}$, XIANGLING YANG ${ }^{1-3}$, AIKICHI IWAMOTO ${ }^{6}$ and HUANLIANG LIU ${ }^{1-3}$
}

\begin{abstract}
${ }^{1}$ Guangdong Institute of Gastroenterology and The Sixth Affiliated Hospital; ${ }^{2}$ Guangdong Provincial Key Laboratory of Colorectal and Pelvic Floor Diseases, Sun Yat-sen University, Guangzhou, Guangdong 510655; ${ }^{3}$ Institute of Human Virology, Sun Yat-sen University, Guangzhou, Guangdong 510080; ${ }^{4}$ Guangdong Provincial Key Laboratory of Allergy and Immunology,

Guangzhou Medical University, Guangzhou, Guangdong 511436; ${ }^{5}$ State Key Laboratory of Oncology in South China, Sun Yat-sen University Cancer Center, Guangzhou, Guangdong 510080, P.R. China; ${ }^{6}$ Advanced Clinical Research Center, Institute of Medical Science, University of Tokyo, Tokyo 108-8639, Japan
\end{abstract}

Received September 9, 2014; Accepted January 23, 2015

DOI: 10.3892/ol.2015.3371

\begin{abstract}
Piperlongumine (PPLGM), an alkaloid isolated from the long pepper (Piper longum L.), can selectively trigger cancer cell death in colorectal cancer cells. The present study investigated whether the c-Jun $\mathrm{NH}_{2}$-terminal kinase (JNK) signaling pathway is involved in PPLGM-induced apoptosis in the human colorectal cancer HCT116 cell line. The results demonstrated that PPLGM reduced the cell viability and induced cell apoptosis in a time- and concentration-dependent manner, without a significant effect on cell cycle distribution. Meanwhile, treatment with $10 \mu \mathrm{M}$ PPLGM resulted in JNK activation within $1 \mathrm{~h}$, and a marked and sustained increase in c-Jun phosphorylation in the HCT116 cells. In addition, SP600125, a general inhibitor of JNK, inhibited PPLGM-induced apoptosis in the HCT116 cells by inhibiting PPLGM-induced c-Jun phosphorylation. Altogether, it can be concluded that the JNK signaling pathway, at least in part, is involved in PPLGM-mediated apoptosis in HCT116 cells.
\end{abstract}

\section{Introduction}

Colorectal cancer (CRC) is one of the leading causes of cancer-related mortality worldwide (1). Nearly half of patients newly diagnosed with colorectal cancer will develop metastases and require systemic chemotherapy (2). As current

Correspondence to: Professor Huanliang Liu, Guangdong Institute of Gastroenterology and The Sixth Affiliated Hospital, Sun Yat-sen University, 26 Yuancun Er Heng Road, Guangzhou, Guangdong 510655, P.R. China

E-mail: huanliang.liu@gmail.com

${ }^{*}$ Contributed equally

Key words: piperlongumine, colorectal cancer, c-Jun $\mathrm{NH}_{2}$-terminal kinases, apoptosis, phosphorylation, SP600125 chemotherapeutic drugs for colorectal cancer have clinically significant toxicities, it is a challenging endeavor to develop novel chemotherapeutic agents with good efficacy and selectivity (3).

Piperlongumine (PPLGM) is a bioactive component that was first isolated from Piper longum L., commonly referred to as the long pepper (4). Traditionally, PPLGM has been used for treating gastrointestinal and respiratory diseases (5). Notably, PPLGM has been shown to selectively target a wide spectrum of cancer cells (6-9). PPLGM induces cancer cell death by triggering different pathways, including apoptosis, necrosis and autophagy (10-13). The elevation of reactive oxygen species (ROS), characteristic of oxidative stress, is an important mechanism by which PPLGM induces cancer-selective cell death $(7,8)$. In addition, ROS-independent mechanisms, such as cellular cross-linking events, may also contribute to the induction of apoptosis by PPLGM (14). Extensive research has documented the fact that PPLGM induces apoptosis in cancer cells by interfering with redox and ROS homeostatic regulators $(8,15)$. The outstanding efficiency of PPLGM in inducing cancer cell death and its low toxicity favor the potential development of this compound as a chemotherapeutic agent against cancer (8).

The c-Jun $\mathrm{NH}_{2}$-terminal kinases (JNKs) are protein kinases that can phosphorylate the c-Jun transcription factor at serine (Ser)63 and -73, resulting in the robust induction of c-Jun transactivation (16). Evidence has accumulated demonstrating that JNKs are involved in a variety of cell activities, including cell apoptosis, which is an important process for tumor suppression $(17,18)$. Further studies have revealed that JNKs play an essential role in cancer cell death induced by redox chemotherapeutic agents $(19,20)$. In particular, PPLGM-mediated oxidative stress has been shown to be partially induced by inhibiting glutathione S-transferase $\pi-1$ (GSTP1), which is a direct negative regulator of JNK, resulting in cancer cell death $(8,21)$. We thus hypothesize that PPLGM induces cell death in colorectal cancer cells via activation of the JNK signaling pathway. The present study investigates the effects of PPLGM on JNK signal transduction and the role of the JNK signaling pathway on PPLGM-mediated cell apoptosis in HCT116 cells. 


\section{Materials and methods}

Reagents. PPLGM and SP600125 (Sigma-Aldrich, St. Louis, MO, USA) were dissolved in dimethyl sulphoxide (DMSO) to a $50 \mathrm{mM}$ solution and stored at $-20^{\circ} \mathrm{C}$. Rabbit monoclonal antibodies (Abs) against c-Jun and phospho-c-Jun at Ser63 were purchased from Epitomics (Burlingame, CA, USA). Rabbit monoclonal Abs against cysteinyl aspartate-specific proteinase-3 (caspase-3) and JNK, and mouse monoclonal Abs against phospho-JNK at Thr183/Tyr185 were obtained from Cell Signaling Technology, Inc., (Beverly, MA, USA). Mouse monoclonal Abs against poly(adenosine diphosphate-ribose) polymerase (PARP) were obtained from BD Biosciences (San Jose, CA, USA). Mouse monoclonal Abs against $\beta$-actin, and anti-mouse immunoglobulin $\mathrm{G}$ and anti-rabbit immunoglobulin $\mathrm{G}$ horseradish peroxidase-conjugated secondary antibodies were purchased from Proteintech Group, Inc. (Chicago, IL, USA).

Cell culture. Human epithelial colorectal adenocarcinoma HCT116 cells were purchased from Culture Collection of Chinese Academy of Science (Shanghai, China) and cultured in RPMI 1640 medium (Gibco Life Technologies, Carlsbad, CA, USA) supplemented with $10 \%$ inactivated fetal bovine serum, $100 \mathrm{IU} / \mathrm{ml}$ penicillin and $100 \mu \mathrm{g} / \mathrm{ml}$ streptomycin in a humidified atmosphere of $5 \% \mathrm{CO}_{2}$ at $37^{\circ} \mathrm{C}$ until confluence.

Cell viability assay. An MTS assay (CellTiter $96^{\circledR}$ AQueous One Solution Cell Proliferation Assay; Promega Corporation, Madison, WI, USA) was used to test cell viability. A total of $3 \times 10^{4} / \mathrm{ml}$ cells in $100 \mu \mathrm{l}$ cell culture medium were incubated with increasing concentrations of PPLGM for $72 \mathrm{~h}$. Detailed description of the indicated duration periods and doses is provided in Fig. 1. Control cells received DMSO for a final concentration that was the identical to the highest concentration of PPLGM, but less than $0.1 \%(\mathrm{v} / \mathrm{v})$. At $4 \mathrm{~h}$ prior to culture termination, $20 \mu \mathrm{l}$ MTS was added to the wells. The absorbance density was read on a 96-well plate reader (Mithras LB 940 Multimode Microplate Reader; Berthold Technologies GmbH \& Co. KG, Bad Wildbad, Baden-Württemberg, Germany). at wavelength of $490 \mathrm{~nm}$.

Trypan blue dye exclusion assay. The HCT116 cells were seeded into 24-well plates to $20-30 \%$ confluency, and then treated with PPLGM in various concentrations for the indicated duration. Detailed description of the indicated duration periods and doses is provided in Fig. 2. The cells were then trypsinized and stained with $0.4 \%$ (w/v) trypan blue (Sigma-Aldrich) and the viable cells were counted using a hematocytometer (Qiujing Biology Company Limited, Shanghai, China).

Cell cycle analysis by flow cytometry. Subsequent to being exposed to a fixed dose of PPLGM for various periods of time, the HCT116 cells were collected and fixed overnight in $66 \%$ cold ethanol at $4^{\circ} \mathrm{C}$. A detailed description of the indicated duration periods and doses is provided in Fig. 3. The cells were then washed twice in cold phosphate-buffered saline (PBS) and labeled with propidium iodide (PI; BD Biosciences, Franklin Lakes, NJ, USA). Cell cycle distribution was determined using a BD FACScanto II flow cytometry analyzer equipped with BD FACSDiva software, version 6.1.3 (BD Biosciences, Franklin Lakes, NJ, USA).

Analysis of cell apoptosis by flow cytometry. Apoptosis was determined by flow cytometry using an Annexin V-fluoroisothiocyanate (FITC)/PI double-staining kit (Nanjing KeyGen Biotechnology, Co., Ltd., Nanjing, Jiangsu, China). The HCT116 cells were incubated with increasing concentration of drugs for the indicated times. A detailed description of the indicated duration periods and doses is provided in Fig. 4. Following the drug treatment, the cells were collected, washed and stained in working solution ( $500 \mu \mathrm{l}$ binding buffer with $5 \mu \mathrm{l}$ Annexin V-FITC and $5 \mu \mathrm{l} \mathrm{PI}$ ) for $15 \mathrm{~min}$ in the dark. Apoptotic cells were then determined by flow cytometry, and the results were analyzed by BD FACSDiva software version 6.1.3.

Western blotting. The procedures for western blotting were performed as described previously (22). Briefly, equal amounts of protein were separated by $10 \%$ sodium dodecyl sulfate-polyacrylamide gel electrophoresis and transferred to a polyvinylidene difluoride membrane (Millipore, Billerica, MA, USA). The membranes were then blocked with $5 \%$ skimmed-milk, $20 \mathrm{mM}$ PBS and $0.1 \%$ Tween-20 buffer for $1 \mathrm{~h}$ at room temperature, and incubated with rabbit anti-c-Jun (dilution, 1:1,000), rabbit anti-phospho-c-Jun at Ser63 (dilution, 1:1,000), rabbit anti-caspase-3 (dilution, 1:1,000), mouse anti-PARP (dilution, 1:1,000), mouse anti- $\beta$-actin (dilution, $1: 10,000)$ and rabbit anti-JNK (dilution, 1:1,000), mouse anti-phospho-JNK at Thr183/Tyr185 (dilution, 1:1,000) overnight at $4^{\circ} \mathrm{C}$. The next day, following incubation with horseradish peroxidase-conjugated secondary antibodies (dilution, 1:10,000) for $1 \mathrm{~h}$ at room temperature, the signals were detected using an enhanced chemiluminescence detection kit (Santa Cruz Biotechnology, Inc., Dallas, TX, USA).

Treatment with JNK inhibitors. The cells were preincubated with the JNK inhibitor, SP600125 $(10 \mu \mathrm{M})$, for $2 \mathrm{~h}$ and then treated with PPLGM $(10 \mu \mathrm{M})$ for $24 \mathrm{~h}$. Cell apoptosis was analyzed by flow cytometry and the protein levels were measured by western blotting.

Statistical analysis. All experiments were performed at least three times, and results are expressed as the mean \pm standard deviation. Statistical analysis was performed by one-way analysis of variance followed by Tukey's test using GraphPad Prism 6.02 software (GraphPad Software, Inc., La Jolla, CA, USA). $\mathrm{P}<0.05$ was considered to indicate a statistically significant difference.

\section{Results}

PPLGM causes concentration- and time-dependent growth inhibition of HCT116 cells. The dose-response effects of PPLGM on HCT116 were evaluated by MTS assay (Fig. 1). The half maximal inhibitory concentration value for PPLGM was $4.6 \mu \mathrm{M}$ in the HCT116 cells at $72 \mathrm{~h}$. Next, the cytotoxic effects of PPLGM on the HCT116 cells were tested by trypan-blue dye exclusion assay (Fig. 2). PPLGM at different concentrations inhibited the cell viability in the HCT116 cells, and the cellular response to the drug increased markedly as the drug 


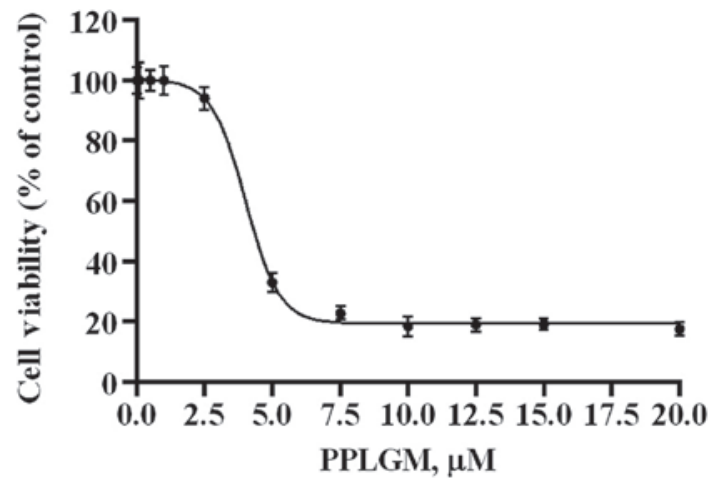

Figure 1.Piperlongumine (PPLGM) reduces the cell viability of HCT116 cells. The HCT116 cells were exposed to the indicated concentrations of PPLGM for $72 \mathrm{~h}$ and then subjected to an MTS proliferation assay. Data are presented as the mean \pm standard deviation from three experiments.

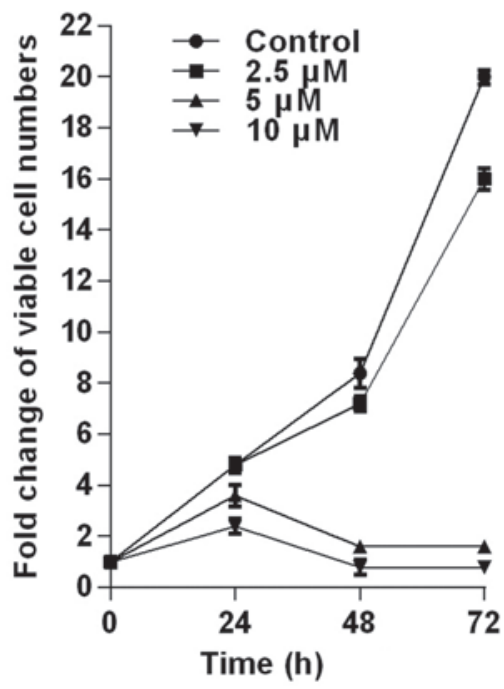

Figure 2. Piperlongumine (PPLGM) inhibits HCT116 cell proliferation in a dose- and time-dependent manner. An equal number of HCT116 cells were plated and cultured in 24-well plates for $24 \mathrm{~h}$, treated with different concentrations of PPLGM and incubated for the indicated time intervals. Cells were then stained with trypan blue and the viable cells were counted. Each experiment was performed in triplicate and the number of viable cells was plotted graphically with the standard deviation.

concentration was raised from 2.5 to $10 \mu \mathrm{M}$ (Fig. 2). The result showed that the inhibition of cell proliferation by PPLGM in the HCT116 cells was concentration- and time-dependent. After the cells had been treated with $5 \mu \mathrm{M}$ PPLGM for $24 \mathrm{~h}$, the cell viability decreased sharply compared with the control (Fig. 2). In addition, flow cytometric analysis revealed no significant alteration in cell-cycle distribution in the HCT116 cells incubated with $5 \mu \mathrm{M}$ PPLGM (Fig. 3). Thus, PPLGM inhibited the growth of HCT116 cells without significantly affecting the cell cycle, suggesting that the loss of viability in the HCT116 cells may be attributable to cell death, but not to cell cycle withdrawal.

PPLGM induces apoptosis in HCT116 cells. To confirm the capability of PPLGM in inducing apoptosis, the control and PPLGM-treated HCT116 cells were assessed by flow cytometry subsequent to staining with Annexin V and PI. After

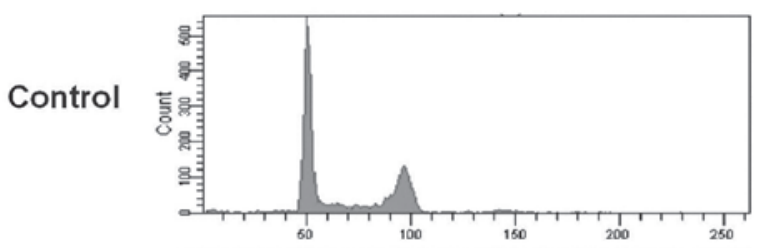

$12 \mathrm{~h}$

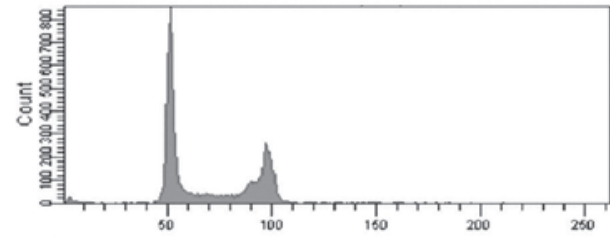

24 h

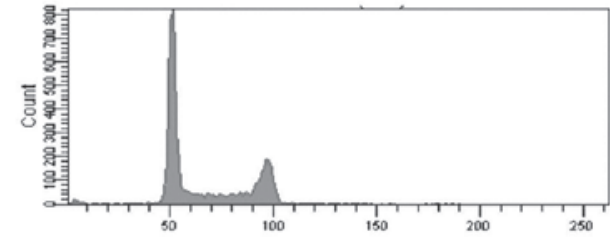

$48 \mathrm{~h}$
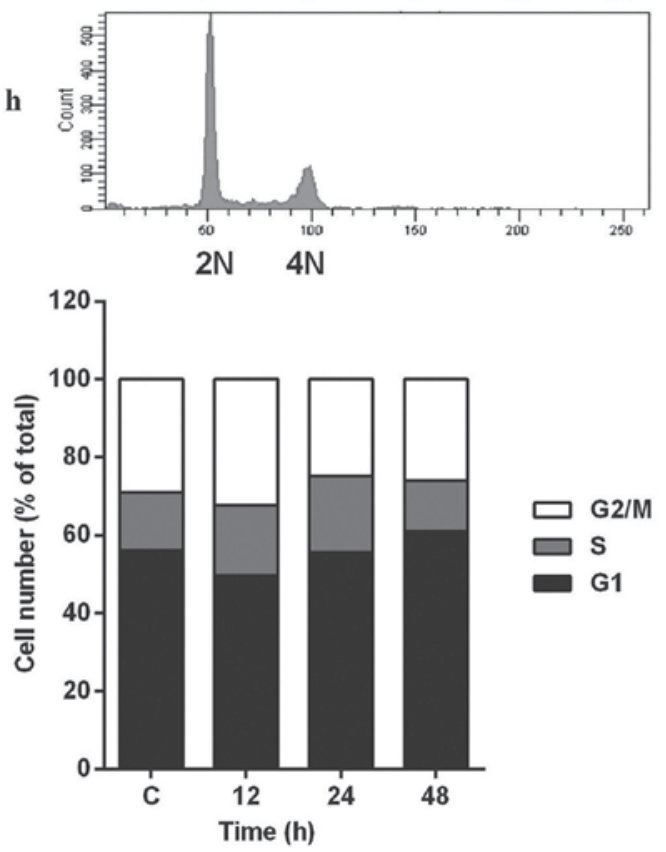

Figure 3. Effect of piperlongumine (PPLGM) on the distribution of cell cycle phases in HCT116 cells. The HCT116 cells were exposed to $5 \mu \mathrm{M}$ PPLGM for the indicated time intervals and analyzed by flow cytometry following staining with propidium iodide. Figures were selected as representative data from three independent experiments. $2 \mathrm{~N}$, diploid cells; $4 \mathrm{~N}$, tetraploid cells.

$48 \mathrm{~h}$ of treatment with $10 \mu \mathrm{M}$ PPLGM, the population of apoptotic HCT116 cells reached $67.3 \%$ (Fig. 4A). These data supported the occurrence of apoptosis in the HCT116 cells following PPLGM treatment. Next, to further verify apoptotic induction, cleaved PARP and caspase- 3 in the PPLGM-treated HCT116 cells were monitored by western blotting. As illustrated in Fig. 4B, PARP cleavage and the reduction of caspase-3 protein levels, known hallmarks of apoptosis, appeared after $24 \mathrm{~h}$ of treatment with $10 \mu \mathrm{M}$ PPLGM. Taken together, these results show that PPLGM induces a substantial level of apoptosis in HCT116 cells.

PPLGM activates the JNK signaling pathway in HCT116 cells. As JNK is the protein kinase that can phosphorylate c-Jun at Ser63 and -73, c-Jun phosphorylation at site Ser63 was selected 
A

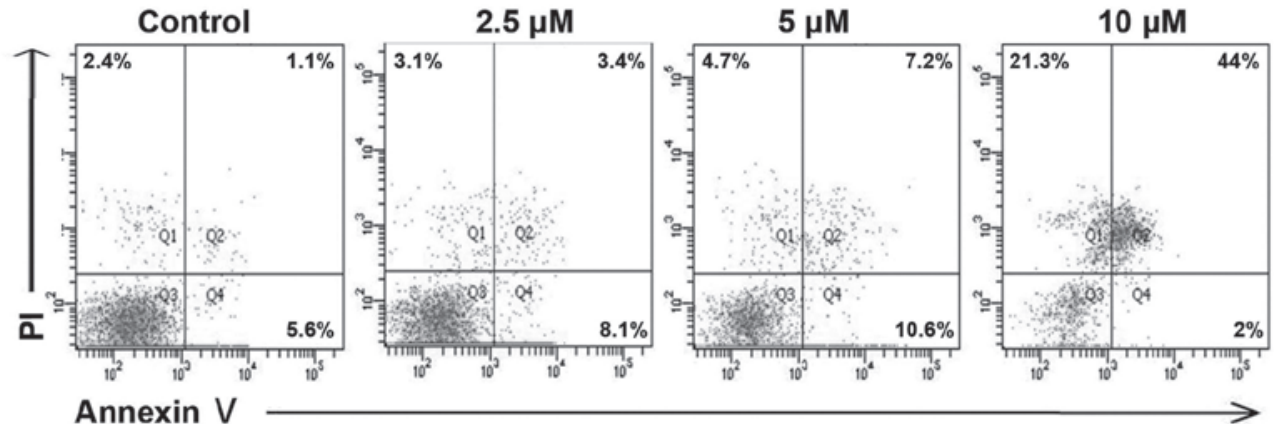

B
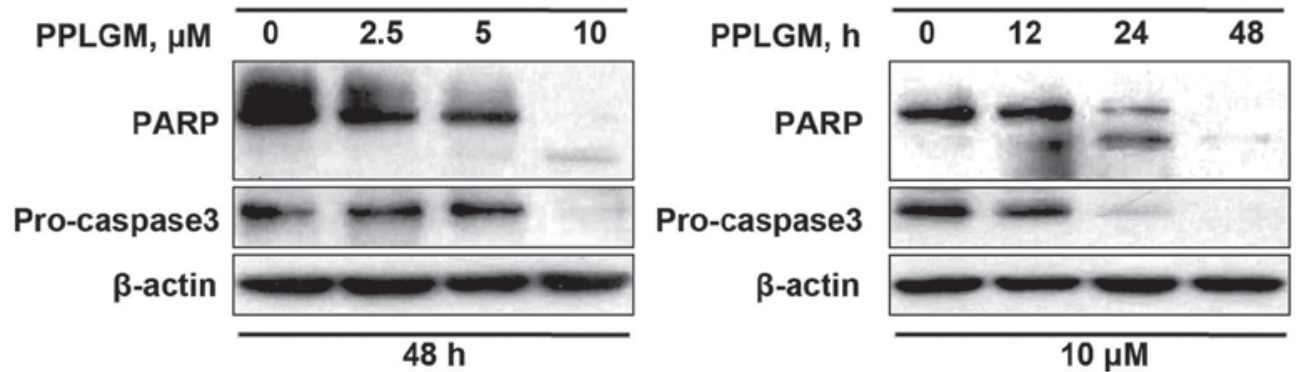

Figure 4. Piperlongumine (PPLGM) triggers apoptosis in HCT116 cells. (A) The cells were treated with PPLGM at increasing concentrations for $48 \mathrm{~h}$ and then underwent Annexin V/propidium iodide (PI) staining for flow cytometric analysis of cell apoptosis. (B) The cells were exposed to PPLGM at increasing concentrations for $48 \mathrm{~h}$ or were treated with $10 \mu \mathrm{M}$ PPLGM for the indicated times. PARP and caspase-3 protein levels were determined by western blotting. $\beta$-actin was used to verify equal loading. Figures were selected as representative data from three independent experiments. PARP, poly(adenosine diphosphate-ribose) polymerase.

A

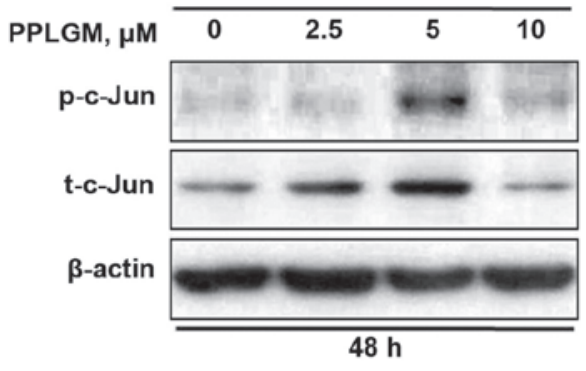

B

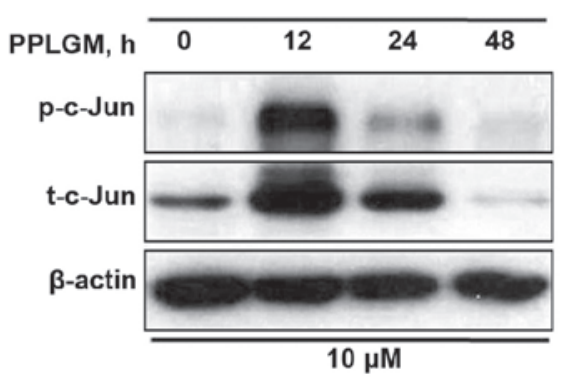

Figure 5. Piperlongumine (PPLGM) promotes c-Jun phosphorylation at Ser63. Protein expression levels were determined by western blotting. $\beta$-actin was used to verify equal loading. (A) HCT116 cells were exposed to PPLGM at increasing concentrations for $48 \mathrm{~h}$. (B) HCT116 cells were treated with $10 \mu \mathrm{M}$ PPLGM for the indicated times. Figures were selected as representative data from three independent experiments. p-, phosphorylated; t-, total.

for evaluation of the JNK signaling pathway. When the cells were incubated with increasing concentrations of PPLGM for $48 \mathrm{~h}$, a moderate increase of c-Jun phosphorylation was detected at $5 \mu \mathrm{M}$ only (Fig. 5A). According to the results of previous studies $(8,9)$ and the current results (Fig. 4), $10 \mu \mathrm{M}$ PPLGM induced cancer

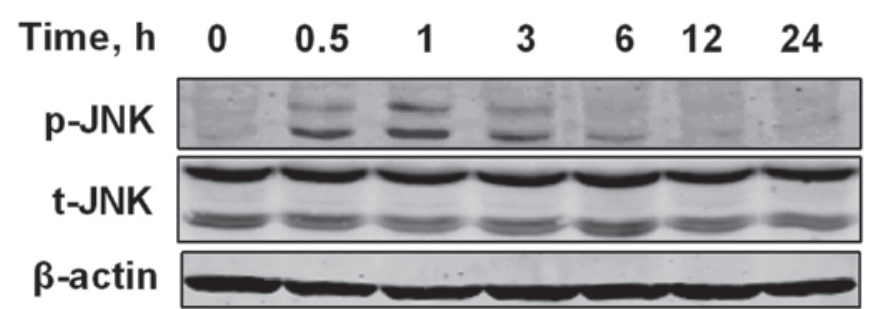

Figure 6. Piperlongumine (PPLGM) induces c-Jun NH2-terminal kinase (JNK) phosphorylation. Protein expression levels were determined by western blotting. $\beta$-actin was used to verify equal loading. HCT116 cells were exposed to $10 \mu \mathrm{M}$ PPLGM for various periods of time. Figures were selected as representative data from three independent experiments. p-, phosphorylated; t-, total.

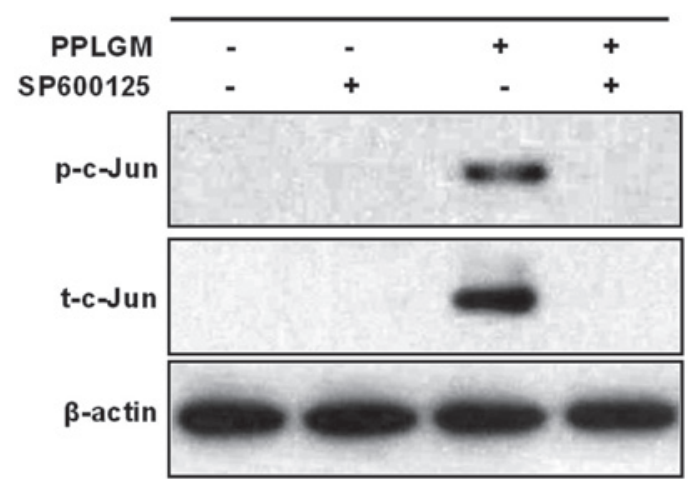

Figure 7. SP600125 inhibits piperlongumine (PPLGM)-induced c-Jun phosphorylation. For the inhibition experiments, the cells were incubated for $2 \mathrm{~h}$ in the presence or absence of $20 \mu \mathrm{M}$ SP600125, then $10 \mu \mathrm{M}$ PPLGM was added and incubated for $24 \mathrm{~h}$. p-c-Jun and t-c-Jun was determined by western blotting. $\beta$-actin was used to verify equal loading. Figures were selected as representative data from three independent experiments. p-, phosphorylated; t-, total. 

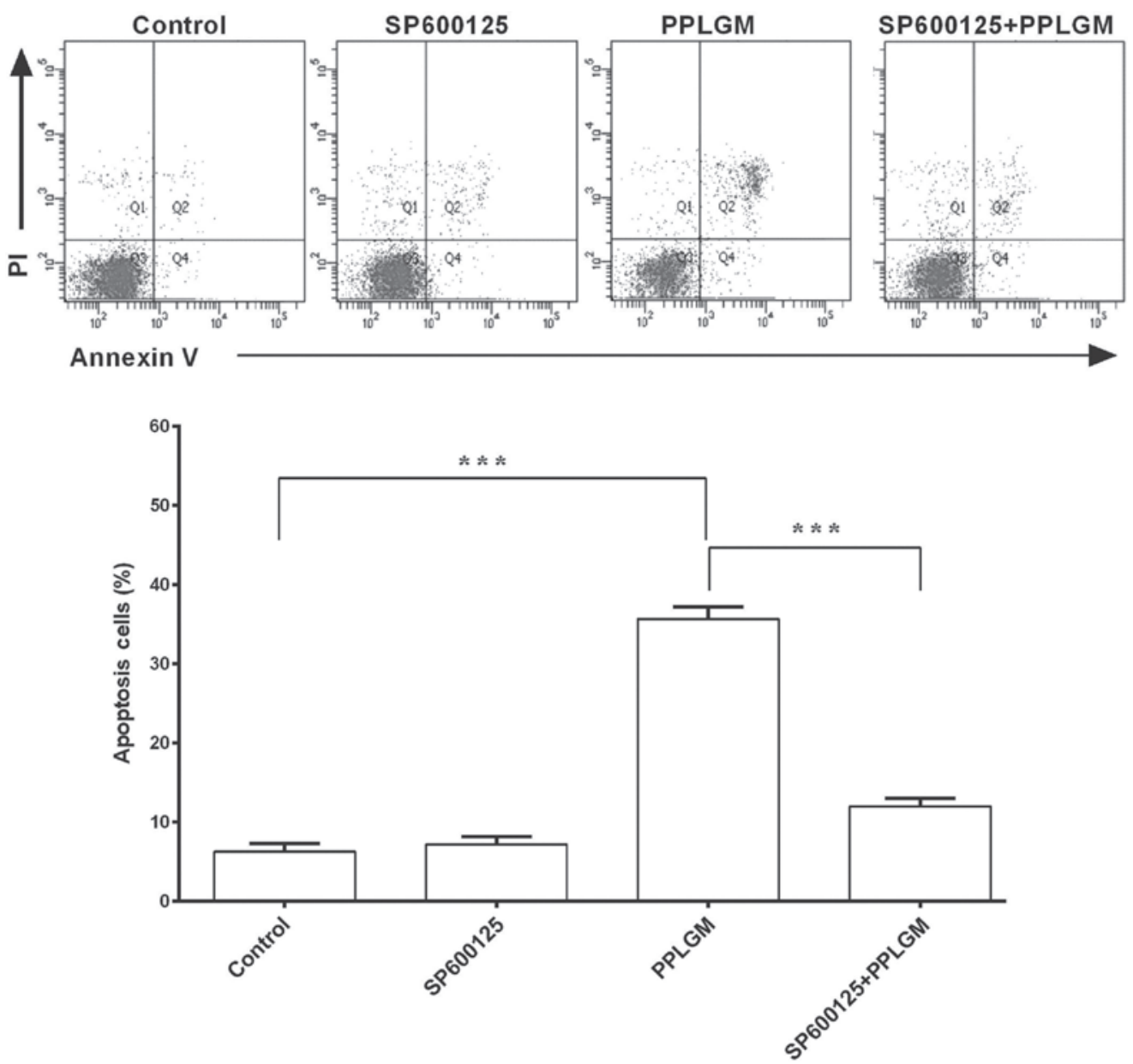

Figure 8. SP600125 rescues HCT116 cells from apoptosis induced by piperlongumine (PPLGM). For the inhibition experiments, the cells were incubated for $2 \mathrm{~h}$ in the presence or absence of $20 \mu \mathrm{M} \mathrm{SP} 600125$, then $10 \mu \mathrm{M}$ PPLGM was added and incubated for $24 \mathrm{~h}$. The induction of apoptosis was estimated by flow cytometric analysis. Each value is the mean \pm standard deviation of three determinations. ${ }^{* * *} \mathrm{P}<0.0001$. PI, propidium iodide.

cell death more significantly than $5 \mu \mathrm{M}$ PPLGM. Therefore, the concentration of $10 \mu \mathrm{M}$ PPLGM was used in subsequent experiments. Treatment with $10 \mu \mathrm{M}$ PPLGM for $12 \mathrm{~h}$ resulted in a marked increase in c-Jun phosphorylation in the HCT116 cells when the cell lysates were immunoblotted with the appropriate phospho-specific antibodies (Fig. 5B). The 10- $\mu$ M PPLGM treatment resulted in sustained c-Jun phosphorylation, which was still apparent after $24 \mathrm{~h}$ in the HCT116 cells (Fig. 5B). In addition, the c-Jun expression level was elevated in parallel with increased c-Jun phosphorylation in the PPLGM-treated HCT116 cells (Fig. 5). The 10- $\mu$ M PPLGM treatment induced c-Jun phosphorylation prior to PARP cleavage and loss of cell viability, suggesting that the JNK signal pathway may mediate PPLGM-induced apoptosis.

To further examine whether the JNK signaling pathway is activated by PPLGM in HCT116 cells, the cells were exposed to $10 \mu \mathrm{M}$ PPLGM for various periods of time, and analyzed for PPLGM-induced changes in JNK phosphorylation by western blot analysis using dual phospho-specific JNK antibodies. As shown in Fig. 6, JNK phosphorylation levels increased in the HCT116 cells within $1 \mathrm{~h}$ of PPLGM incubation and then decreased again from $3 \mathrm{~h}$ onwards.

SP600125 inhibits PPLGM-mediated apoptosis through restraining JNK signal pathway activation in HCT116 cells.
To identify whether the JNK signaling pathway was involved in PPLGM-mediated apoptosis, SP600125, a general inhibitor of JNK, was co-incubated with PPLGM in the HCT116 cells and cell apoptosis was then determined using flow cytometry. Pre-treatment with the SP600125 JNK inhibitor completely blocked the PPLGM-induced phosphorylation of c-Jun (Fig. 7) and significantly inhibited PPLGM-induced cell apoptosis in the HCT116 cells (Fig. 8). The percentage of apoptotic cells following treatment with $20 \mu \mathrm{M}$ SP600125, $10 \mu \mathrm{M}$ PPLGM, or $20 \mu \mathrm{M}$ SP600125 plus $10 \mu \mathrm{M}$ PPLGM were $7.2,35.7$ and $12.0 \%$, respectively. The difference between PPLGM alone and PPLGM plus SP600125 was significant $(\mathrm{P}<0.0001)$.

\section{Discussion}

Recent data has showed that PPLGM can selectively kill various cancer cells, including human colorectal cancer cells $(8,9)$. To the best of our knowledge, the present study is the first to indicate that the JNK signaling pathway is involved in PPLGM-induced cell apoptosis in human colorectal cancer cells. The results showed that PPLGM reduced cell viability independently of cell cycle withdrawal and induced cell death in a time- and concentration-dependent manner. At the same time, JNK signaling was activated during PPLGM treatment in the HCT116 cells. In addition, SP600125 
inhibited PPLGM-induced JNK signaling and apoptosis in the HCT116 cells, suggesting that PPLGM-mediated apoptosis was at least partially dependent on the activation of the JNK signal pathway in the HCT116 cells (23).

Accumulated data have shown that PPLGM induces cell death through different pathways in multiple types of cancer cells (7-12). Previous studies have demonstrated that caspase-3-mediated PARP cleavage and cell cycle arrest at the $\mathrm{G}_{2} / \mathrm{M}$ phase are involved in PPLGM-induced cell apoptosis in human prostate cancer PC-3 cells (11). In the present study, HCT116 cells treated with PPLGM demonstrated likewise up-regulation of PARP/procaspase-3 cleavage (Fig. 4B), but not cell cycle arrest (Fig. 3); this discrepancy in the effect of PPLGM on cell cycle distribution may be a result of the different responses to PPLGM in different cell lines.

c-Jun, a cognate substrate for JNK, is a labile protein that is degraded by JNK under non-stressed conditions (24). However, a variety of cellular stresses, such as oxidative stress, can strongly activate the JNKs, which inhibit c-Jun ubiquitination and promote c-Jun transcription through c-Jun phosphorylation $(24,25)$. Inhibition of the ubiquitin-proteasome system induced by PPLGM in cancer cells may also reduce the ubiquitin-dependent degradation of c-Jun (26). Collectively, a steady elevation of c-Jun expression is associated with the expression and stabilization of c-Jun. Hence, in the present study, it followed that sustained c-Jun phosphorylation was concomitant with the c-Jun overexpression observed during PPLGM treatment (Fig. 5).

The positive association between c-Jun activation and cell apoptosis has been well documented in neurons, endothelial and myeloma cells, fibroblasts and colorectal cancer cells $(3,27-30)$. Similarly, the activation of JNK and the subsequent phosphorylation of c-Jun have been linked with apoptotic cell death induced by PPLGM in HCT116 cells (Figs. 7 and 8). Conversely, other studies have shown that the inhibition of JNK by SP600125 sensitizes tumor cells to CD95-induced apoptosis in HCT116 cells and that this effect is cell line-specific (31). Whether c-Jun activation leads to the inhibition or promotion of apoptosis should be dependent on the stimuli and the cell type.

Recent data have indicated that the mitogen-activated protein kinase (MAPK) core pathways are involved in PPLGM-induced cancer cell death. Notably, p38 MAPK activation leads to cell death through autophagy in human osteosarcoma U2OS cells (12). The present results showed that JNK signaling is involved in PPLGM-induced apoptosis in HCT116 cells (Figs. 7 and 8). As reported earlier in the colorectal cancer HT-29 cell line (9), the extracellular signal-regulated kinase (ERK) signaling pathway is also activated in PPLGM-treated HCT116 cells (data not shown), strongly arguing that a decrease in ERK and an increase in JNK are required for the induction of apoptosis (17). A previous study in hamster fibroblast CC139 cells demonstrated that phosphoinositide 3'-kinase (PI3K) inhibition is necessary for JNK-mediated cell death (32). In addition, PPLGM causes PI3K inhibition to induce caspase-dependent apoptosis in human triple-negative breast cancer cells (13). Altogether, the mechanisms of signal transduction are complicated during the course of PPLGM-induced cancer cell death and the cross-talk between different signaling pathways should be further elucidated.
In summary, the present results suggested that in the HCT116 cells, PPLGM reduced cell viability and triggered cell apoptosis through the JNK signal pathway in a concentration- and time-dependent manner. A clear understanding of the molecular mechanisms of PPLGM-mediated cell apoptosis may shed light on the further clinical development of PPLGM for chemotherapy.

\section{Acknowledgements}

The authors would like to thank Dr Xinhui Fu, Dr Zihuan Yang, Dr Jun Hu and Ms. Zhiting Chen for providing technical assistance. This study was supported by Sun Yat-sen University '100 Talents Program', the Guangdong Innovative Research Team Program (grant no. 2009010058), the Science and Information Technology Bureau of Guangzhou, Guangdong (grant no. 2011J5200009), the Guangdong Provincial Department of Science and Technology (grant no. 2012B050500004), Overseas Excellent Professor Project, and the Japanese Ministry of Education, Culture, Sports, Science and Technology, Program of Japanese Initiative for Global Research Network on Infectious Diseases.

\section{References}

1. Siegel R, Ma J, Zou Z and Jemal A: Cancer statistics, 2014. CA Cancer J Clin 64: 9-29, 2014.

2. Van Cutsem E, Köhne CH, Hitre E, et al: Cetuximab and chemotherapy as initial treatment for metastatic colorectal cance. N Engl J Med 360: 1408-1417, 2009.

3. Ham J, Babij C, Whitfield J, et al: A c-Jun dominant negative mutant protects sympathetic neurons against programmed cell death. Neuron 14: 927-939, 1995.

4. Chatterjee A and Dutta CP: Alkaloids of Piper longum Linn. I. Structure and synthesis of piperlongumine and piperlonguminine. Tetrahedron 23: 1769-1781, 1967.

5. Bezerra DP, Pessoa C, de Moraes MO, Saker-Neto N, Silveira ER and Costa-Lotufo LV: Overview of the therapeutic potential of piplartine (piperlongumine). Eur J Pharm Sci 48: 453-463, 2013.

6. Han SS, Son DJ, Yun H, Kamberos NL and Janz S: Piperlongumine inhibits proliferation and survival of Burkitt lymphoma in vitro. Leuk Res 37: 146-154, 2013.

7. Liu JM, Pan F, Li L, et al: Piperlongumine selectively kills glioblastoma multiforme cells via reactive oxygen species accumulation dependent JNK and p38 activation. Biochem Biophys Res Commun 437: 87-93, 2013.

8. Raj L, Ide T, Gurkar AU, et al: Selective killing of cancer cells by a small molecule targeting the stress response to ROS. Nature 475: 231-234, 2011.

9. Randhawa H, Kibble K, Zeng H, Moyer MP and Reindl KM: Activation of ERK signaling and induction of colon cancer cell death by piperlongumine. Toxicol In Vitro 27: 1626-1633, 2013.

10. Bezerra DP, Militão GC, de Castro FO, et al: Piplartine induces inhibition of leukemia cell proliferation triggering both apoptosis and necrosis pathways. Toxicol In Vitro 21: 1-8, 2007.

11. Kong EH, Kim YJ, Cho HJ, et al: Piplartine induces caspase-mediated apoptosis in PC-3 human prostate cancer cells. Oncol Rep 20: 785-792, 2008.

12. Wang Y, Wang JW, Xiao X, et al: Piperlongumine induces autophagy by targeting p38 signaling. Cell Death Dis 4: e824, 2013.

13. Shrivastava S, Kulkarni P, Thummuri D, et al: Piperlongumine, an alkaloid causes inhibition of PI3K/Akt/mTOR signaling axis to induce caspase-dependent apoptosis in human triple-negative breast cancer cells. A Internat J Program Cell Death 19: 1148-1164, 2014.

14. Adams DJ, Dai M, Pellegrino G, et al: Synthesis, cellular evaluation, and mechanism of action of piperlongumine analogs. Proc Natl Acad Sci USA 109: 15115-15120, 2012. 
15. Kim TH, Song J, Kim SH, et al: Piperlongumine treatment inactivates peroxiredoxin 4 , exacerbates endoplasmic reticulum stress, and preferentially kills high-grade glioma cells. Neuro Oncol 16: 1354-1364, 2014

16. Kyriakis JM and Avruch J: Mammalian MAPK signal transduction pathways activated by stress and inflammation: a 10-year update. Physiol Rev 92: 689-737, 2012.

17. Xia Z, Dickens M, Raingeaud J, Davis RJ and Greenberg ME: Opposing effects of ERK and JNK-p38 MAP kinases on apoptosis. Science 270: 1326-1331, 1995.

18. Chang L and Karin M: Mammalian MAP kinase signalling cascades. Nature 410: 37-40, 2001.

19. Hu R, Kim BR, Chen C, Hebbar V and Kong AN: The roles of JNK and apoptotic signaling pathways in PEITC-mediated responses in human HT-29 colon adenocarcinoma cells. Carcinogenesis 24: 1361-1367, 2003.

20. Xu C, Shen G, Yuan X, et al: ERK and JNK signaling pathways are involved in the regulation of activator protein 1 and cell death elicited by three isothiocyanates in human prostate cancer PC-3 cells. Carcinogenesis 27: 437-445, 2006.

21. Wang T, Arifoglu P, Ronai Z and Tew KD: Glutathione S-transferase P1-1 (GSTP1-1) inhibits c-Jun N-terminal kinase (JNK1) signaling through interaction with the $\mathrm{C}$ terminus. J Biol Chem 276: 20999-21003, 2001

22. Song M, Chen D, Lu B, et al: PTEN loss increases PD-L1 protein expression and affects the correlation between PD-L1 expression and clinical parameters in colorectal cancer. PLoS One 8 : e65821, 2013

23. Bennett BL, Sasaki DT, Murray BW, et al: SP600125, an anthrapyrazolone inhibitor of Jun N-terminal kinase. Proc Natl Acad Sci USA 98: 13681-13686, 2001.
24. Fuchs SY, Dolan L, Davis RJ and Ronai Z: Phosphorylation-dependent targeting of c-Jun ubiquitination by Jun N-kinase. Oncogene 13: 1531-1535, 1996.

25. Musti AM, Treier M and Bohmann D: Reduced ubiquitin-dependent degradation of c-Jun after phosphorylation by MAP kinases. Science 275: 400-402, 1997.

26. Jarvius M, Fryknäs M, D'Arcy P, Sun C, Rickardson L, Gullbo J, Haglund C, Nygren P, Linder S and Larsson R: Piperlongumine induces inhibition of the ubiquitin-proteasome system in cancer cells. Biochem Biophys Res Commun 431: 117-123, 2013.

27. Wang N, Verna L, Hardy S, et al: c-Jun triggers apoptosis in human vascular endothelial cells. Circ Res 85: 387-393, 1999.

28. Podar K, Raab MS, Tonon G, et al: Up-regulation of c-Jun inhibits proliferation and induces apoptosis via caspase-triggered c-Abl cleavage in human multiple myeloma. Cancer Res 67: 1680-1688, 2007.

29. Bossy-Wetzel E, Bakiri L and Yaniv M: Induction of apoptosis by the transcription factor c-Jun. EMBO J 16: 1695-1709, 1997.

30. Teraishi F, Wu S, Zhang L, et al: Identification of a novel synthetic thiazolidin compound capable of inducing c-Jun NH2-terminal kinase-dependent apoptosis in human colon cancer cells. Cancer Res 65: 6380-6387, 2005 .

31. Kuntzen C, Sonuc N, De Toni EN, et al: Inhibition of c-Jun-N-terminal-kinase sensitizes tumor cells to CD95-induced apoptosis and induces G2/M cell cycle arrest. Cancer Res 65: 6780-6788, 2005.

32. Molton SA, Todd DE and Cook SJ: Selective activation of the c-Jun N-terminal kinase (JNK) pathway fails to elicit Bax activation or apoptosis unless the phosphoinositide 3-kinase (PI3K) pathway is inhibited. Oncogene 22: 4690-4701, 2003. 